\title{
A Candidate for the Attentional Bottleneck: Set-size Specific Modulation of the Right TPJ during Attentive Enumeration
}

\author{
Petra Vetter*, Brian Butterworth, and Bahador Bahrami
}

\begin{abstract}
Several recent behavioral studies have shown that the enumeration of a small number of items (a process termed subitizing) depends on the availability of attentional resources and is not a preattentive process as previously thought. Here we studied the neural correlates of visual enumeration under different attentional loads in a dual-task paradigm using fMRI. Relatively intact subitizing under low attentional load compared to impaired subitizing under high attentional load was associated with an increase in BOLD signal in the right temporo-parietal junction (rTPJ). Crucially, attentionally modulated response in the rTPJ was specific to
\end{abstract}

\section{INTRODUCTION}

Perceiving the number of a small set of items, up to about three or four, subjectively appears to be an effortless task. However, there has been considerable controversy in the scientific literature as to whether the enumeration of small sets (so-called subitizing) requires visual attention. Many earlier studies have argued that subitizing occurs preattentively and in parallel, either based on flat behavioral performance curves (Simon \& Vaishnavi, 1996; Sagi \& Julesz, 1984), preserved subitizing ability in patients with attentional deficits (Vuilleumier \& Rafal, 2000; Dehaene \& Cohen, 1994) or the absence of brain activations in attentional networks (Piazza, Giacomini, Le Bihan, \& Dehaene, 2003; Sathian et al., 1999). Preattentive subitizing has traditionally been thought of as a functionally distinct process from attentive and serial "counting," that is, the enumeration of five and more items.

Several recent behavioral studies, however, demonstrated that subitizing can be severely compromised when visual attention is engaged elsewhere. Subitizing performance is markedly impaired in the attentional blink (Egeth, Leonard, \& Palomares, 2008; Olivers \& Watson, 2008) and under conditions of inattention or divided attention in an in-

University College London, UK

*Current address: Centre for Cognitive Neuroimaging, Department of Psychology, University of Glasgow, 58 Hillhead Street, Glasgow G12 8QB, UK, or via e-mail: p.vetter@psy.gla.ac.uk. small set sizes (up to 3 items) and did not occur at larger set sizes (5-7 items). This result has two implications: (1) Subitizing involves part of the fronto-parietal network for stimulus-driven attention providing neural evidence against preattentive subitizing. (2) Activity in rTPJ is set-size modulated. Together with similar evidence from studies probing visual short-term memory, this result suggests that rTPJ modulation might reflect the brain's ability to attentively handle small set sizes. Thus, the rTPJ may play an important role for the emergence of a capacity limit in both enumeration and visual short-term memory.

attentional blindness paradigm (Railo, Koivisto, Revonsuo, \& Hannula, 2008). By manipulating attentional load in a dual-task paradigm, we showed that the more attentional resources are withdrawn from an enumeration task, the more enumeration performance is impaired, both within as well as outside the subitizing range (Vetter, Butterworth, \& Bahrami, 2008). Furthermore, given that subitizing performance improves by increasing stimulus presentation times beyond 50 msec, Poiese, Spalek, and DiLollo (2008) argued that subitizing must benefit from feedback processing from higher-level brain areas beyond V1. Taken together, this evidence severely challenges the claim of a preattentive subitizing mechanism.

Most previous imaging studies failed to find specific activations for subitizing but succeeded in revealing higherlevel activations in parietal and frontal areas for counting (Piazza et al., 2003; Piazza, Mechelli, Butterworth, \& Price, 2002; Sathian et al., 1999). In most cases (Piazza et al., 2003; Sathian et al., 1999), this was again interpreted as consistent with an attentional dichotomy in enumeration: Preattentive subitizing involving only visual cortex (Sathian et al., 1999) and attentive counting recruiting attentional networks (Piazza et al., 2003). However, the chosen baseline conditions with which the subitizing conditions were compared might have been suboptimal, either because they involved tasks equally effortless as subitizing [such as color naming (Piazza et al., 2003) or single target detection (Sathian et al., 1999)] or because they might have elicited implicit subitizing due to the presence of one object. Similarly, 
the attentional activations related to effortful counting are quite expected when contrasted to relatively effortless subitizing. That is, previous studies did not control for the increasing attentional demands with increasing number of items. Thus, the failure to find subitizing-related activations in attentional brain networks is insufficient evidence for preattentive subitizing.

In this study, we investigated the neural correlates of visual enumeration under direct manipulation of the attentional resources allocated to an enumeration task. We employed the same dual-task paradigm, which we developed previously in a behavioral study (Vetter et al., 2008). We varied the attentional load of a primary target detection task (no load, low load, or high load), and thus, gradually withdrew attentional resources from a secondary enumeration task. Under low attentional load, subjects detected a simple feature in a centrally presented diamond shape (see Figure 1A for a stimulus example). Under high attentional load, subjects detected two specific color-orientation conjunctions. As a secondary task, subjects enumerated high-contrast grating targets in a ring surrounding the central diamond shape, choosing between numerosities 1,3 , 5, and 7 . Under single task, participants performed only the enumeration task.

This paradigm had the following advantages:

(1) The load manipulation allowed us to directly compare the neural correlates of enumeration when attentional resources were restricted (i.e., under high load) to those situations when they were less restricted (low load) or unrestricted (no load), both for set sizes within the subitizing range (numerosities 1 and 3 ) and outside the subitizing range (numerosities 5 and 7 , hence referred to as the estimation range). It is important to keep in mind that we investigated the neural corre- lates of the attentional modulation of enumeration rather than the neural correlates of enumeration per se.

(2) Our dual-task paradigm was well suited to circumvent the problem of suboptimal baseline conditions. Load theory (Lavie, 1995, 2005) asserts that under low attentional load, neural resources can be automatically allocated to distractor processing, that is, the secondary task. Under high attentional load, however, distractor processing is suppressed as most neural resources have to be dedicated to the primary task (Lavie, 1995, 2005). In the case of the current paradigm, this implies that under single task (no load) or low load, sufficient neural resources are available to process numerosity, whereas under high load, neural resources are entirely dedicated to the primary task and numerosity processing is suppressed (as indeed suggested by impaired behavioral performance, c.f. Vetter et al., 2008). Thus, the high load condition provided a more adequate baseline condition than those employed in previous studies.

(3) Our experimental design allowed us to dissociate the brain regions involved in attentional effort (i.e., those related to increased task demands due to the attentional load of the primary task) from numerosity processing areas that are attentionally modulated. Thus, this design controls more appropriately for attentional effects of increasing processing demands with increasing numerosity than previous designs.

We predicted that if subitizing is truly preattentive and recruits only low-level processing, then comparing intact or mildly impaired subitizing to severely impaired subitizing [contrast subitizing (low load) > subitizing (high load)] should either not yield any specific difference in BOLD signal, or only in early visual areas such as primary visual cortex. If subitizing is attentive, however, the same contrast

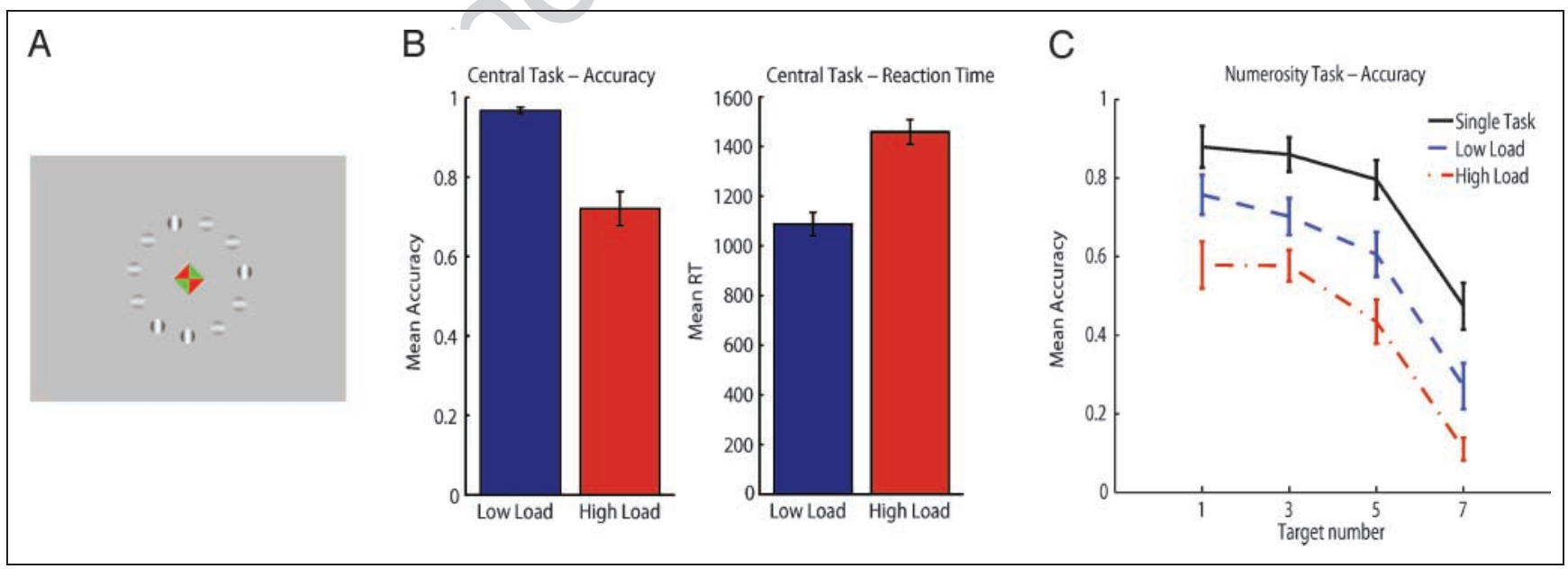

Figure 1. (A) Stimulus example. Under dual-task conditions, subjects first detected a certain color target in the center (low load: any red; high load: green right-tilted or yellow left-tilted triangle combinations) and then enumerated the number of high contrast, vertically oriented targets in the circle $(1,3,5$, or 7$)$. Under the single-task condition, subjects only performed the enumeration task. (B) Behavioral results for the primary target detection task that incorporated the manipulation of attention load. Mean accuracy (left) and mean reaction times (right) are plotted for both low and high load condition. (C) Accuracy (proportion correct) of the secondary enumeration task as a function of target number. Error bars indicate 1 standard error of the mean (SEM). 
was predicted to result in responses in higher-level attentional areas such as parietal and frontal cortex (Corbetta \& Shulman, 2002). More specifically, associated BOLD response should be modulated by numerosity if it is specific to enumeration and not purely caused by task-independent suppression of secondary task processing.

In line with load theory (Lavie, 2005) and evidence from previous studies employing attentional load manipulations (Schwartz et al., 2005; Pinsk, Doniger, \& Kastner, 2004), we predicted that the high load condition should engage the fronto-parietal attentional network more than the low load condition, and accordingly, the dual-task conditions should engage the attentional network more than the single-task condition. Thus, we predicted that the effect of attentional effort and dual task (e.g., by applying the contrast high load $>$ low load and the contrast low load $>$ single task) would result in increased activation in such networks.

\section{METHODS}

\section{Participants}

Eighteen healthy adults (mean age: 24.5 years, range: 1935, 10 women) participated in the experiment. Subjects were screened for right-handedness, intact color vision, and MRI suitability, that is, the absence of neurological or psychiatric illnesses and absence of metal in the body. All signed informed consent and were paid for their participation. The study was approved by the ethics committee of the Department of Psychology at UCL.

\section{Stimuli}

Briefly, the stimulus set consisted of a central colored diamond shape $\left(1.6^{\circ}\right)$ and a surrounding circle of hard-edged Gabor patches $\left(3.3^{\circ}\right)$, with each patch subtending $1^{\circ}$. Gabor patches were either vertically oriented, high-contrast (100\%) targets or horizontally oriented, low-contrast (50\%) distractors (see example stimulus in Figure 1A). All other aspects of the stimulus were the same as in Vetter et al. (2008). Stimuli were generated using the Cogent toolbox (www.vislab.ucl.ac.uk/Cogent/) for MATLAB (Mathworks, Inc, Natick, MA).

\section{Task and Experimental Procedure}

We employed a dual-task paradigm. The primary target detection task was the same as in Vetter et al. (2008). Under low attentional load, subjects detected a simple feature in the central diamond shape (the color red, independent of spatial arrangement). Under high attentional load, subjects performed a color-orientation conjunction task: They indicated the presence of either two green triangles oriented along the right-tilted diagonal or two yellow triangles along the left-tilted diagonal. Subjects were instructed to discriminate these conjunctions from the opposite combinations. The secondary numerical task required subjects to judge whether the circle contained one, three, five, or seven high-contrast targets. The total number of items in the circle ranged from 9 to 12 , counterbalanced for each target number and load condition. Note that neither the total number of items in the circle, nor the number of distractors, reliably correlated with target number. The use of Gabor patches ensured that overall luminance was constant across all numerosities. Three experimental conditions were employed: single numerosity task (no load), dual task (low load), and dual task (high load).

Each experimental block started with a screen displaying the instruction for the load condition ("Number only" for single task, "Red" for low load, "Green right-tilted or yellow left-tilted" for high load). In each trial, a fixation cross was displayed for $1 \mathrm{sec}$, followed by the stimulus for 200 msec and, subsequently, followed by a mask which stayed on the screen until response. Note that short stimulus presentation times prevented verbal counting. Subjects were given a maximum of $2.5 \mathrm{sec}$ to respond to the primary task and a maximum of $3.5 \mathrm{sec}$ to respond to the secondary task. The next trial started two repetition times (7.2 sec) following the subject's response.

A right-hand keypad with four response buttons was used. Under dual-task conditions, subjects responded always first to the primary task with the index and middle fingers (indicating target presence or absence, respectively) and, subsequently, to the secondary numerical task using all four buttons (index finger to little finger), indicating target numbers 1, 3, 5, and 7. Single-task conditions required only one response to the numerical task. Speed was emphasized for the primary task and accuracy for the secondary task. Subjects received training with the task prior to scanning. The scanning session lasted for about 1 hour.

\section{Experimental Design}

The fMRI design was blocked with respect to attentional load (3 levels) and event-related with respect to numerosity ( 4 levels). Data were collected in 4 runs of 6 blocks ( 2 for each load condition) of 16 trials each, giving rise to 384 trials in total, 32 trials for each of the 12 Numerosity $\times$ Load combinations. The order of load conditions was counterbalanced within each run. Numerosity levels were counterbalanced across the 2 blocks of each experimental condition in each run and the order of numerosity levels was randomized.

\section{Image Acquisition}

Functional images measuring BOLD signal were acquired in a Siemens TIM Avanto 1.5-Tesla MRI scanner at the Birkbeck-UCL Neuroimaging Centre. A gradient-echo EPI sequence was employed $[38$ slices, repetition time $(\mathrm{TR})=$ $3.6 \mathrm{sec}$, echo time $(\mathrm{TE})=50 \mathrm{msec}$, field of vision $(\mathrm{FOV})=$ $192 \times 192$, matrix $=64 \times 64$ ], giving a notion resolution of $3 \times 3 \times 3 \mathrm{~mm}$. Between 230 and 300 volumes for each run 
were recorded (depending on how quickly individual subjects responded to the task). Reduced signal was recorded in the frontal lobe due to failure of the frontal head coil in some scanning sessions.

\section{Data Analysis}

The behavioral data were analyzed as in Vetter et al. (2008). The imaging data were analyzed using statistical parametric mapping (SPM5; www.fil.ion.ucl.ac.uk/spm/). Functional images were submitted to a random effects group analysis using a general linear model according to standard SPM processing (Friston et al., 1995). The first five volumes of each run were discarded. All other volumes were spatially realigned to the first image of the first run, time-corrected with respect to the middle slice and "unwarped" to remove movement-related variance. Scans were normalized to a standard anatomical MNI template (resampled voxel size: $2 \times 2 \times 2 \mathrm{~mm}^{3}$ ), smoothed with an isotropic 8-mm FWHM Gaussian kernel, and high-pass filtered (1/120 Hz cutoff).

In the subject-level analysis, the 12 conditions of interest (3 load conditions $\times 4$ numerosity levels) were modeled by stick functions convolved with a canonical hemodynamic response function. Statistical parametric maps were generated from linear contrasts testing the main effects in each subject and then included in a group analysis using a $3 \times 4$ ANOVA. Main results are reported at $p=.05$ with familywise error (FWE) correction for multiple comparison at the voxel level. In cases when no significant activations were found on the corrected level, the threshold was lowered to $p=.0001$ or $p=.001$ (uncorrected), and the limited strength of those results was taken into account when interpreting the data. Activation clusters of less than 10 voxels are not reported. At some selected regions of interest, small-volume correction was applied by selecting a spherical space of $10 \mathrm{~mm}$ around the region of interest and adjusting $p$ values to this volume.

\section{RESULTS}

\section{Behavioral Results}

\section{Primary Target Detection Task (Figure 1B)}

Mean accuracy and reaction time data for the primary target detection task are depicted in Figure 1B for both load conditions. Subjects were significantly less accurate $[t(17)=$ $5.82, p<.001]$ and significantly slower $[t(17)=-14.76$, $p<.001$ ] under high load compared to low load. Thus, the manipulation of attentional load was effective.

\section{Secondary Numerosity Judgement Task (Figure 1C)}

Mean accuracy of the secondary enumeration task is plotted in Figure $1 \mathrm{C}$ as a function of target number. As expected, accuracy decreased with target number $[F(3,51)=60.91$, $p<.001]$. Note that the task was designed to cover the full range of enumeration accuracy from near ceiling to near floor across the different levels of task difficulties. As such, it was predicted to observe ceiling performance in the easiest task condition (numerosity 1, single task) and floor performance in the hardest task condition (numerosity 7 , high load). More importantly, with respect to the manipulation of attentional load, subjects enumerated significantly less accurately under low load compared to single task, and even less accurately under high load compared to low load [repeated measures ANOVA: main effect $F(2$, $34)=78.60, p<.001$; all post hoc comparisons $p<.001$, Bonferroni-corrected]. No interaction between load condition and target number was observed $[F(6,102)=.89$, $p>.05]$.

\section{Imaging Results}

Having established the behavioral effect of attentional load on enumeration performance in the subitizing and estimation range, we turned to the fMRI data to identify the neural correlates of the attentional modulation of enumeration. According to our hypothesis, the primary interest lay in comparing activations for intact or mildly impaired subitizing (under single task or low load) with activations for severely impaired subitizing (under high load). Following load theory (Lavie, 2005), activity levels related to the secondary task (i.e., enumeration) were expected to be more suppressed under high load compared to low load or single task. Thus, we first focused on the contrasts low load $>$ high load and single task $>$ high load, both within the subitizing range (numerosities 1 and 3) and the estimation range (numerosities 5 and 7), to establish the effect of load modulated numerosity processing.

\section{Effect of Attentionally Modulated Numerosity Processing}

Contrast low load > bigh load. When considering selectively the subitizing range (numerosities 1 and 3), we found one significant activation in the right temporoparietal junction (rTPJ)/right superior temporal gyrus at MNI coordinates [40 -5422] for the contrast subitizing (low load) $>$ subitizing (high load) $(Z=5.12, p=.005$, FWE corrected; Figure $2 \mathrm{~A}$ ). In order to assess the pattern of rTPJ activity across numerosity ranges, the estimated BOLD signal change in the peak voxel (beta weights) was extracted for each subject in each condition and the group average is plotted in Figure 2B. Note that the "baseline" level at 0 is a consequence of the conventions of the general linear model in SPM and, therefore, negative values do not necessarily reflect suppression below resting state. Our experimental design did not include a resting baseline. In line with load theory (Lavie, 2005), we focused on the relative difference between activation levels. rTPJ activity was modulated by numerosity in all load conditions. Activity decreased with increasing numerosity $[F(3,51)=4.26$, $p<.01]$ in the single-task condition and in the low load 


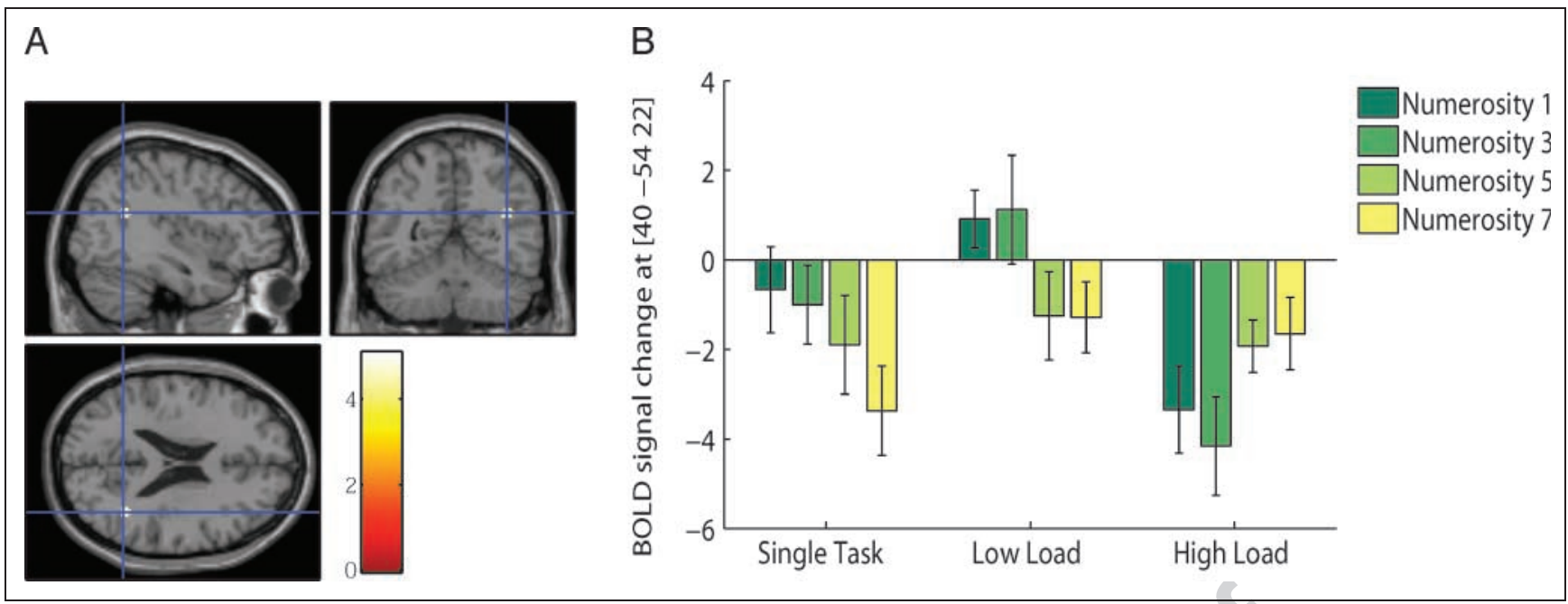

Figure 2. (A) Subitizing specific activation at the rTPJ [ $40-5422]$ as revealed by the contrast subitizing (low load) $>$ subitizing (high load). The color scale indicates $Z$ value. (B) BOLD signal change (beta weights; arbitrary units) at the rTPJ for each level of attentional load and numerosity. Note that the baseline level at 0 is a consequence of the conventions of the general linear model in SPM and, therefore, negative values do not necessarily reflect suppression below resting state. The relative difference between activation levels is the meaningful comparison here.

condition $[F(3,51)=2.83, p<.05]$, but increased in the high load condition $[F(3,51)=3.15, p<.05]$.

The response in the rTPJ was specific to the subitizing range. When considering selectively the estimation range [numerosities 5 and 7, contrast estimation (low load) > estimation (high load)], no significant activations were found throughout the whole brain, even at reduced thresholds (up to $p=.01$, uncorrected). Also applying smallvolume correction for this contrast at the rTPJ location identified by the subitizing contrast did not yield any significant response.

Given the differential results for the subitizing and the estimation range, we also tested for the interaction effect between numerosity range and attentional load [contrast (subitizing $>$ estimation $) \times$ (low load $>$ high load) , which yielded the same right TPJ activation as found in the subitizing specific contrast (MNI coordinates [ $40-54$ 22], $Z=3.28, p=.001$, uncorrected). This confirmed that the load modulation of the rTPJ was specific to the low numerosities.

When considering the whole numerosity range (contrast low load $>$ high load), no activations were found on the corrected level, but lowering the threshold yielded responses in the rTPJ, bilateral cingulate gyrus, right subparietal sulcus, right inferior parietal lobule, and corpus callosum (see Table 1 in Supplemental Material). The fact that the rTPJ response in the interaction and across the whole numerosity range was only visible at reduced thresholds suggests that it was mainly driven by the subitizingrelated response.

Contrast single task $>$ bigh load. This comparison incorporated the largest possible difference between enumeration in the absence of load and enumeration under high load, thus including both the effect of dual task and attentional load on enumeration. Activations specific to this contrast were only found at the uncorrected level $(p<$ .0001), mainly in bilateral cingulate cortex, right middle temporal and middle occipital gyri, right inferior parietal lobule, right thalamus, left angular gyrus, and left insula (see Table 2 in Supplementary Material). Some areas were also activated in the subitizing selective contrast [subitizing (single task) > subitizing (high load)], although activations in the estimation selective contrast [estimation (single task) $>$ estimation (high load)] either did not reach significance or have sufficient cluster sizes ( $>10$ voxels).

\section{Effect of Magnitude}

Contrasts subitizing > estimation and estimation > subitizing. Here activations for small and large numerosities were compared with each other. Neither the contrast subitizing $>$ estimation nor estimation $>$ subitizing yielded any significant response at thresholds as low as $p=.001$ (uncorr.). The same was true when each load condition was analyzed separately. This suggests that the above mentioned interaction effect was not driven by an overall differential response to small or large numerosities per se, but by a load-induced modulation across set size.

\section{Effect of Dual Task and Attentional Effort}

Contrasts high load > low load, low load > single task, and high load $>$ single task. Here, the opposite contrasts to those applied for numerosity processing were employed. Note that there is a crucial conceptual difference between the comparisons low load or single task $>$ high load and the comparisons high load $>$ low load or single task. In line with load theory (Lavie, 2005), the former tested the attentional modulation of the secondary task, whereas the latter tested the effect of the primary task demands. Contrast high load $>$ low load revealed the 
effects of increased attentional effort due to increased load, whereas the contrast low load $>$ single task related to the effect of dual-task situations. The contrast high load $>$ single task incorporated both effects. The effect of attentional effort (high load $>$ low load) was overall weaker than the effect of dual task or both combined [effects significant at $p<.0001$ (uncorr.) compared to the contrasts high load $>$ single task or low load $>$ single task, significant at $p<.05$, FWE corrected]. A widespread network of activations was found mainly in occipital cortex (left lingual gyrus, right precuneus) and limbic structures (bilateral parahippocampal gyrus and right cingulate cortex) as well as activations in the right superior parietal lobule, bilateral superior temporal gyrus, right-lateralized frontal areas (inferior frontal gyrus, postcentral gyrus), and several midbrain and cerebellar structures. Results are summarized in Table 3 (Supplementary Material).

\section{DISCUSSION}

The retrieval of small numerosity information from a visual scene has, for a long time, been thought of as occurring in an automatic and preattentive manner (Piazza et al., 2003; Vuilleumier \& Rafal, 2000; Sathian et al., 1999; Dehaene \& Cohen, 1994; Sagi \& Julesz, 1984). Given recent behavioral evidence against this claim (Egeth et al., 2008; Olivers \& Watson, 2008; Poiese et al., 2008; Railo et al., 2008; Vetter et al., 2008), we investigated the attentional modulation of subitizing on the neural level. Our design was motivated by the load theory of attentional selection (Lavie, 1995, 2005), and thus, bore the advantage of (1) providing an adequate baseline condition under which subitizing related activity was suppressed and (2) the possibility to dissociate attentionally modulated numerosity processing from the effect of attentional effort.

Enumeration of small set sizes (1 and 3) under low attentional load compared to enumeration under high attentional load resulted in increased BOLD response in the rTPJ. Crucially, no differential BOLD response was found during the attentionally modulated enumeration of numerosities exceeding the subitizing range (5 and 7). The rTPJ has been implicated as an important part of a rightlateralized ventral fronto-parietal network responsible for stimulus-driven, bottom-up attentional control (Corbetta \& Shulman, 2002). The rTPJ is predominantly involved in target detection and reorienting attention toward salient or unexpected sensory events (e.g., Serences et al., 2005; Corbetta, Kincade, Ollinger, McAvoy, \& Shulman, 2000; Marois, Leung, \& Gore, 2000), particularly when these events are behaviorally relevant (Kincade, Abrams, Astafiev, Shulman, \& Corbetta, 2005). More specifically, the rTPJ is deactivated during monitoring of behaviorally irrelevant stimuli (distractors) and becomes active once a relevant target (contained within a prespecified target set) appears (Shulman et al., 2003), implicating a possible role of the rTPJ in the filtering of behaviorally relevant stimuli. Our results, in light of load theory (Lavie, 2005), are in line with this role: Under high attentional load, rTPJ activity is suppressed as attentional resources are allocated entirely to the primary task. Processing of secondary task stimuli is severely compromised (as evident in the behavioral performance), which is in accord with the idea of an rTPJ "filter" successfully eliminating numerical processing. Under low attentional load, the rTPJ is less suppressed and behaviorally relevant secondary task targets (i.e., numerical stimuli) can be detected more successfully. It should be noted, however, that this interpretation is an adaptation of the original filter hypothesis as we did not compare between detection of task-relevant and taskirrelevant stimuli but between tasks with different levels of load (being always task-relevant but graded with respect to the allocation of processing resources). This implies the assumption that under high load, secondary task stimuli are treated by the brain as more "irrelevant" (due to the restriction of processing resources) than under low load.

The crucial and novel aspect in our result is that attentionally modulated rTPJ response is restricted to the small set sizes ( 1 and 3 ) and is not present at larger set sizes ( 5 and 7). This precludes the occurrence of rTPJ activation solely due to the increased processing of secondary task stimuli under low load or due to the relatively unexpected appearance of the stimulus on the screen. Furthermore, the analysis of BOLD signal change at the peak voxel in the rTPJ (Figure 2B) clearly indicates that rTPJ activity is modulated by the number of items that are enumerated. This is an important aspect of the data because it relates rTPJ activity to a numerosity-modulated brain process rather than an unspecific process of, for example, load-related task demands.

Interestingly, Todd, Fougnie, and Marois (2005) found that suppression in the rTPJ (at a slightly more lateralized site in the supramarginal gyrus) is modulated by the number of items to be memorized in a change detection task probing visual short-term memory. rTPJ suppression was stronger at set size 3 than at set size 1 , but did not differ between set sizes 3 and 6 . Thus, it appears that modulation of rTPJ activity occurs at small set sizes (1 to $3)$ and ceases at larger set sizes, both in cases where sets are either memorized (Todd et al., 2005) or enumerated (this study). Given that both visual short-term memory as well as enumeration are characterized by a capacity limit at around set size 4 (although this capacity limit may not be fixed, cf. Bays \& Husain, 2008; Alvarez \& Cavanagh, 2004), Cowan (2001) proposed that both processes might be restricted by the same attentional bottleneck. Our results, together with those by Todd et al. (2005), suggest that the rTPJ may be one of the prime sites of such an attentional bottleneck. This idea also ties in with the hypothesis of the rTPJ acting as a filter: Reacting to behaviorally relevant targets while suppressing irrelevant distractors (Shulman, Astafiev, McAvoy, d'Avossa, \& Corbetta, 2007; Shulman et al., 2003), with the extension that this filter function is restricted to small set sizes of items. 
The rTPJ is also part of the brain's default mode network (Shulman et al., 2007), which is generally activated during passive rest and deactivated during active task performance (for a review, see Buckner, Andrews-Hanna, \& Schacter, 2008). However, the default network has never been investigated in the light of dual-task conditions or manipulations of attentional load. It is thus rather speculative to relate the default mode theory to the current data, particularly as we did not include a passive condition. Even if one assumes that default network activations are inversely correlated with task demands, then those activation levels should be higher under single-task conditions than under high load conditions. The contrast single task $>$ high load (Table 2 of Supplementary Material) showed that this is the case for some areas associated with the default network such as the inferior parietal lobule and the cingulate gyrus, but not for the rTPJ. The subitizing specific decrease in rTPJ activation between the low load and the high load condition can therefore not entirely be explained by differential engagement of the default mode network. This is not in contradiction with the filter hypothesis because, as Shulman et al. (2007) point out, the filter hypothesis specifically relates TPJ activations to the "stimulus-driven reorienting to behaviourally relevant stimuli outside the focus of attention" (Shulman et al., 2007, p. 2626) rather than merely to active task performance.

With respect to the attentional requirements of small numerosity judgment, the involvement of the rTPJ in subitizing and, as such, a higher-level area associated with stimulus-driven attention provides further neural evidence against the previous claim of preattentive subitizing (Piazza et al., 2003; Sathian et al., 1999). This is in agreement with a similar finding by Ansari, Lyons, van Eimeren, and Xu (2007), who identified rTPJ activation related to small numerosity judgment (1-4 items) in comparison to much larger numerosity judgment (10-40 items). In contrast to Ansari and colleagues, however, our design incorporated a direct attentional manipulation and allowed the study of numerosity processing under different stages of attentional suppression. Our results thus probed more directly the interaction of attention with enumeration. As predicted, the high load condition provided a more suitable baseline condition than those used in previous studies (e.g., Piazza et al., 2002, 2003; Sathian et al., 1999), as activity in the rTPJ was suppressed under high attentional load compared to the low load condition (see Figure 2B).

When analyzing the whole range of numerosities, activations in similar areas as found in previous enumeration studies were revealed, such as the middle temporal gyrus and the middle occipital gyrus (Piazza et al., 2002; Sathian et al., 1999), as well as the inferior parietal lobule (Ansari et al., 2007; Castelli, Glaser, \& Butterworth, 2006). The latter is also part of the ventral fronto-parietal attention network (Corbetta \& Shulman, 2002), and thus, is ideally suited to mediate between the attentional as well as the numerical task demands. Activity in cingulate cortex, associated with decision processes in general (e.g., Forstmann et al., 2008) and the selection of numerical responses in particular (Bush et al., 1998), ties in well with the activation of the left angular gyrus, which is involved in both number magnitude processing and finger gnosis (Rusconi, Walsh, \& Butterworth, 2005).

Our design furthermore dissociated attentionally modulated numerosity processing from attentional effort, and thus, controlled better for effects of task difficulty than previous studies (e.g., Piazza et al., 2002, 2003; Sathian et al., 1999). As expected, the effects of attentional effort and dual-task activated areas related to visual processing (left lingual gyrus and right precuneus) as well as areas belonging to both the ventral (superior temporal gyrus, inferior frontal gyrus) and the dorsal fronto-parietal attention network (superior parietal lobule) (Corbetta \& Shulman, 2002). This overlaps with findings from other studies employing a manipulation of attentional load (e.g., Schwartz et al., 2005; Pinsk et al., 2004). Strong bilateral activity in the parahippocampal gyrus, often associated with object recognition (e.g., Maguire, Frith, Burgess, Donnett, \& O'Keefe, 1998) and color detection (e.g., Dojat et al., 2006), reflects the task demands of the primary task: detecting certain spatial arrangements of colored triangles involving retrieval from memory.

Given that the rTPJ is involved in many different kinds of attentional processing and is set-size modulated also in change detection tasks, we do not claim that the observed TPJ modulation is specific to numerosity processing [although ironically, some of the studies investigating rTPJ function involved a numerical task, either number detection (Shulman et al., 2003, 2007) or number comparison (Todd et al., 2005)]. We rather suggest that the rTPJ reflects the attentional orienting to behaviorally relevant stimuli and is set-size limited regarding the number of targets that need to be handled. We also do not conclude that rTPJ activity is exclusively specific to the subitizing process and that the enumeration of larger set sizes requires an altogether distinct functional mechanism as some have proposed (Feigenson, Dehaene, \& Spelke, 2004; Piazza et al., 2003; Sathian et al., 1999; Trick \& Pylyshyn, 1994). The conclusion that the judgment of small and large numerosities is subserved by distinct mechanisms cannot be made purely on the basis of a brain area being responsive to one numerosity range but not to the other (an assumption previously made with respect to larger numerosities; see Piazza et al., 2003; Sathian et al., 1999). Indeed, when contrasting small numerosities with large numerosities across load conditions or even within each load condition, no area could be isolated that was preferentially activated by small or large numerosities. The reason why this result differs from previous imaging studies (Piazza et al., 2002, 2003; Sathian et al., 1999) probably lies in the fact that both our experimental design (attentional load manipulation) as well as our stimulus design (use of distractors and decorrelation from luminance) minimized a confounding effect of increasing mental effort with numerosity. 
Despite the set-size modulation of the rTPJ, we would like to emphasize that BOLD signal change at the rTPJ cannot explain the whole behavioral performance pattern. It is also highly likely that other brain areas, not identified in this study, are involved in generating the overall behavioral effect. Particularly for the estimation range this would be a gross overinterpretation, as the rTPJ location was identified using the contrast subitizing (low load) > subitizing (high load). Furthermore, given that behavioral estimation performance in the high load condition is close to floor, the associated brain signals are likely to exhibit a smaller signalto-noise ratio. In light of this, also the rather surprising finding of increasing rTPJ activity with increasing numerosity in the high load condition has to be interpreted with care. However, the comparable level of rTPJ suppression in the estimation range in both low and high load conditions is in line with our interpretation that rTPJ activity is load modulated at small set sizes but not at large set sizes. A result of this differential modulation is a decreasing trend in the low load condition and an increasing trend in the high load condition (cf. Figure 2B).

Two possibilities for the set-size selective modulation in the rTPJ are conceivable: (1) Based on Weber's law, Ross (2003) argued that the resolution of the visual system reaches a numerical discrimination threshold at about four items (i.e., a stimulus difference of roughly 25\%) and that the successful discrimination of higher set sizes requires larger stimulus differences. Thus, the relative difference between five and seven items might be too small to be differentially treated by the visual system, and thus, rTPJ activity is not modulated any more between these higher set sizes. This interpretation implies that there may be a "natural boundary" for set sizes that can be differentially handled by the brain. This would explain why subitizing (or memorizing small sets) is such a seemingly effortless task. (2) Activity in the r'TPJ (or any other area) might also be modulated at higher set sizes, but the relative difference in activity between these stimuli is too small to be detected by the fMRI method or the specific experimental settings employed here. This would, in fact, explain why we did not find significant activations specifically related to the estimation of higher numerosities. However, the fact that Ansari et al. (2007) did not find an rTPJ modulation at higher numerosities when adjusting stimulus differences accordingly argues against such an explanation for the case of the TPJ.

\section{Conclusions}

We identified an area in the rTPJ as implicated in the attentive enumeration of small numerosities. As in studies probing visual short-term memory (Todd et al., 2005), rTPJ modulation was specific for small set sizes and did not detectably occur at larger set sizes. In accordance with its previously associated filter function (Shulman et al., 2003, 2007), the rTPJ might therefore be a prime candidate for a capacity-limited attentional bottleneck in the brain.

\section{Acknowledgments}

We thank Marinella Cappelletti and Cathy Price for help with SPM analysis; Marty Sereno, Joe Devlin, Fred Dick, and Roi CohenKadosh for fruitful discussions; and Geraint Rees for helpful comments on the manuscript. This work was supported by the European Commission (B. Bahrami: Mind Bridge Project; P. Vetter and B. Butterworth: RTN NUMBRA)

Reprint requests should be sent to Petra Vetter, Institute of Cognitive Neuroscience, University College London, 17 Queen Square, London WC1N 3AR, UK, or via e-mail: p.vetter@ucl.ac.uk.

\section{REFERENCES}

Alvarez, G. A., \& Cavanagh, P. (2004). The capacity of visual short-term memory is set both by visual information load and by number of objects. Psychological Science, 15, 106-111.

Ansari, D., Lyons, I. M., van Eimeren, L., \& Xu, F. (2007). Linking visual attention and number processing in the brain: The role of the temporo-parietal junction in small and large symbolic and nonsymbolic number comparison. Journal of Cognitive Neuroscience, 19, 1845-1853.

Bays, P. M., \& Husain, M. (2008). Dynamic shifts of limited working memory resources in human vision. Science, 321, 851-854.

Buckner, R. L., Andrews-Hanna, J. R., \& Schacter, D. L. (2008). The brain's default network: Anatomy, function and relevance to disease. Annals of the New York Academy of Sciences, 1124, 1-38.

Bush, G., Whalen, P. J., Rosen, B. R., Jenike, M. A., McInerney, S. C., \& Rauch, S. L. (1998). The counting Stroop: An interference task specialized for functional neuroimaging-validation study with functional MRI. Human Brain Mapping, 6, 270-282.

Castelli, F., Glaser, D. E., \& Butterworth, B. (2006). Discrete and analogue quantity processing in the parietal lobe: A functional MRI study. Proceedings of the National Academy of Sciences, U.S.A., 103, 4693-4698.

Corbetta, M., Kincade, J. M., Ollinger, J. M., McAvoy, M. P., \& Shulman, G. L. (2000). Voluntary orienting is dissociated from target detection in human posterior parietal cortex. Nature Neuroscience, 3, 292-297.

Corbetta, M., \& Shulman, G. L. (2002). Control of goal-directed and stimulus-driven attention in the brain. Nature Reviews Neuroscience, 3, 201-215.

Cowan, N. (2001). The magical number 4 in short-term memory: A reconsideration of mental storage capacity. Behavioral and Brain Sciences, 24, 87-114; discussion 114-85.

Dehaene, S., \& Cohen, L. (1994). Dissociable mechanisms of subitizing and counting: Neuropsychological evidence from simultanagnosic patients. Journal of Experimental Psychology: Human Perception and Performance, 20, 958-975.

Dojat, M., Piettre, L., Delon-Martin, C., Pachot-Clouard, M., Segebarth, C., \& Knoblauch, K. (2006). Global integration of local color differences in transparency perception: An fMRI study. Visual Neuroscience, 23, 357-364.

Egeth, H. E., Leonard, C. L., \& Palomares, M. (2008). The role of attention in subitizing: Is the magical number 1 ? Visual Cognition, 16, 463-473.

Feigenson, L., Dehaene, S., \& Spelke, E. (2004). Core systems of number. Trends in Cognitive Sciences, 8, 307-314.

Forstmann, B. U., Wolfensteller, U., Derrfuss, J., Neumann, J., Brass, M., Ridderinkhof, K. R., et al. (2008). When the choice is ours: Context and agency modulate the neural bases of decision-making. PLOS ONE, 3, e1899. 
Friston, K. J., Holmes, A. P., Worsley, K. J., Poline, J. B., Frith, C. D., \& Frackowiak, R. S. (1995). Statistical parametric maps in functional imaging: A general linear approach. Human Brain Mapping, 2, 189-210.

Kincade, J. M., Abrams, R. A., Astafiev, S. V., Shulman, G. L., \& Corbetta, M. (2005). An event-related functional magnetic resonance imaging study of voluntary and stimulus-driven orienting of attention. Journal of Neuroscience, 25, 4593-4604.

Lavie, N. (1995). Perceptual load as a necessary condition for selective attention. Journal of Experimental Psychology: Human Perception and Performance, 21, 451-468.

Lavie, N. (2005). Distracted and confused?: Selective attention under load. Trends in Cognitive Sciences, 9, 75-82.

Maguire, E. A., Frith, C. D., Burgess, N., Donnett, J. G., \& O'Keefe, J. (1998). Knowing where things are: Parahippocampal involvement in encoding object locations in virtual large-scale space. Journal of Cognitive Neuroscience, 10, 61-76.

Marois, R., Leung, H. C., \& Gore, J. C. (2000). A stimulus-driven approach to object identity and location processing in the human brain. Neuron, 25, 717-728.

Olivers, C. N. L., \& Watson, D. G. (2008). Subitizing requires attention. Visual Cognition, 16, 439-462.

Piazza, M., Giacomini, E., Le Bihan, D., \& Dehaene, S. (2003). Single-trial classification of parallel pre-attentive and serial attentive processes using functional magnetic resonance imaging. Proceedings of the Royal Society of London, Series B, Biological Sciences, 270, 1237-1245.

Piazza, M., Mechelli, A., Butterworth, B., \& Price, C. J. (2002). Are subitizing and counting implemented as separate or functionally overlapping processes? Neuroimage, 15 , 435-446.

Pinsk, M. A., Doniger, G. M., \& Kastner, S. (2004). Push-pull mechanism of selective attention in human extrastriate cortex. Journal of Neurophysiology, 92, 622-629.

Poiese, P., Spalek, T. M., \& DiLollo, V. (2008). Attentional involvement in subitizing: Questioning the preattentive hypothesis. Visual Cognition, 16, 474-485.

Railo, H., Koivisto, M., Revonsuo, A., \& Hannula, M. M. (2008). The role of attention in subitizing. Cognition, 107, 82-104.

Ross, J. (2003). Visual discrimination of number without counting. Perception, 32, 867-870.

Rusconi, E., Walsh, V., \& Butterworth, B. (2005). Dexterity with numbers: rTMS over left angular gyrus disrupts finger gnosis and number processing. Neuropsychologia, 43, $1609-1624$.

Sagi, D., \& Julesz, B. (1984). Detection versus discrimination of visual orientation. Perception, 13, 619-628.

Sathian, K., Simon, T. J., Peterson, S., Patel, G. A., Hoffman, J. M., \& Grafton, S. T. (1999). Neural evidence linking visual object enumeration and attention. Journal of Cognitive Neuroscience, 11, 36-51.

Schwartz, S., Vuilleumier, P., Hutton, C., Maravita, A., Dolan, R. J., \& Driver, J. (2005). Attentional load and sensory competition in human vision: Modulation of fMRI responses by load at fixation during task-irrelevant stimulation in the peripheral visual field. Cerebral Cortex, 15, 770-786.

Serences, J. T., Shomstein, S., Leber, A. B., Golay, X., Egeth, H. E., \& Yantis, S. (2005). Coordination of voluntary and stimulusdriven attentional control in human cortex. Psychological Science, 16, 114-122.

Shulman, G. L., Astafiev, S. V., McAvoy, M. P., d'Avossa, G., \& Corbetta, M. (2007). Right TPJ deactivation during visual search: Functional significance and support for a filter hypothesis. Cerebral Cortex, 17, 2625-2633.

Shulman, G. L., McAvoy, M. P., Cowan, M. C., Astafiev, S. V., Tansy, A. P., d'Avossa, G., et al. (2003). Quantitative analysis of attention and detection signals during visual search. Journal of Neurophysiology, 90, 3384-3397.

Simon, T. J., \& Vaishnavi, S. (1996). Subitizing and counting depend on different attentional mechanisms: Evidence from visual enumeration in afterimages. Perception $\varepsilon$ Psychophysics, 58, 915-926.

Todd, J. J., Fougnie, D., \& Marois, R. (2005). Visual short-term memory load suppresses temporo-parietal junction activity and induces inattentional blindness. Psychological Science, 16, 965-972.

Trick, L. M., \& Pylyshyn, Z. W. (1994). Why are small and large numbers enumerated differently? A limited-capacity preattentive stage in vision. Psychological Review, 101, 80-102.

Vetter, P., Butterworth, B., \& Bahrami, B. (2008). Modulating attentional load affects numerosity estimation: Evidence against a pre-attentive subitizing mechanism. PLOS ONE, 3, e3269.

Vuilleumier, P. O., \& Rafal, R. D. (2000). A systematic study of visual extinction. Between- and within-field deficits of attention in hemispatial neglect. Brain, 123, 1263-1279. 